\title{
O MÉTODO DA PESQUISA CONVERGENTE ASSISTENCIAL EM UM ESTUDO COM PESSOAS COM DOENÇA ARTERIAL CORONARIANA
}

\author{
Converging assistance research approach in a study with people with coronary arterial \\ disease \\ El método de investigación convergente asistencial en un estudio con personas con \\ enfermedad arterial coronariana
}

Albertina Bonetti

Denise Guerreiro V. da Silva²

Mercedes Trentini ${ }^{3}$

\section{RESUMO}

0 objetivo deste relato foi destacar a relevância do método da Pesquisa Convergente Assistencial (PCA). Toma como referência um estudo que objetivou introduzir inovações no cuidado pela construção, implementação e análise de um Programa de Vivências Corporais Lúdicas com pessoas com Doença Arterial Coronariana. A PCA caracteriza-se pela propriedade de se articular com a prática assistencial em saúde. Desta forma, as ações de assistência vão sendo incorporadas no processo de pesquisa e vice-versa. Os resultados mostraram que a PCA configurou-se como um método apropriado para introduzir inovações na prática de saúde. As pessoas foram beneficiadas com uma melhor compreensão de seus próprios problemas e participaram na construção de um viver melhor com sua condição crônica. 0 estudo também contribuiu para o desenvolvimento do conhecimento teórico referente a formas lúdicas para o cuidado de si e de prevenção de complicações relacionadas à Doença Arterial Coronariana.

Palavras-chave. Pesquisa. Doenças crônicas. Exercício.

\begin{abstract}
The objective of this report is to highlight the relevance of the method of Converging Assistance Research (PCA). It takes as reference a study that aimed to introduce innovations in the context of the practice through the construction, implementation and analysis of a Body Ludic Experience Program with persons diagnosed with coronary arteriosclerosis disease. The PCA approach is characterized by its property of interacting with the health assistance. Accordingly, health actions are gradually incorporated in the research process and vice versa. The findings showed that the PCA consisted of a proper method for introducing innovation in the health practice for self care and for the prevention of the coronary arteriosclerosis disease complications. The study also has contributed for the knowledge development related to self-care and to prevention of coronary atherosclerosis complications.
\end{abstract}

Keywords: Inquiry. Chronic diseases. Exercise.

\section{Resumen}

Enfoca la relevancia del método de Investigación Convergente Asistencial (ICA) que fue utilizada en un estudio que tuvo como objetivo introducir innovaciones en el cuidado por la construcción, implementación y análisis de un Programa de Vivencias Corporales Lúdicas (PVCL) con personas con Enfermedad Arterial Coronariana. La ICA se caracteriza por la propiedad de articularse con la práctica asistencial en salud. De esa forma, las acciones de asistencia van siendo incorporadas en el proceso de investigación y viceversa. Los resultados indicaron que la ICA fue un método apropiado para introducir innovaciones en la práctica de salud. Los enfermos fueron beneficiados con una mejor comprensión de sus problemas y participaron de la construcción de un mejor modo de vivir con su condición crónica. El estudio contribuyó también para el desarrollo del conocimiento teórico referente a las maneras lúdicas para cuidarse y prevenir complicaciones relacionadas a la Enfermedad Arterial Coronariana.

Palabras llave: Investigación. Enfermedades crónicas. Ejercicio.

'Dra. em Enfermagem; professora do Departamento de Educação Física da Universidade Federal de Santa Catarina (UFSC). Integrante do grupo de pesquisa NUCRON. Florianópolis - SC. Brasil. E-mail: tina@cds.ufsc.br; ²Dra. em Enfermagem; professora do Departamento de Enfermagem e do Programa de PósGraduação em Enfermagem da UFSC. Líder do grupo de pesquisa NUCRON. Bolsista de produtividade do CNPq. Florianópolis-SC. Brasil. E-mail: denise@ccs.ufsc.br; ${ }^{3}$ Dra. em Enfermagem; professora aposentada do Departamento de Enfermagem e do Programa de Pós-Graduação em Enfermagem da UFSC. Integrante do grupo de pesquisa NUCRON. Florianópolis - SC. Brasil. E-mail: mertini@terra.com.br. 


\section{INTRODUÇÃO}

Este artigo apresenta o relato de um estudo que incluiu ações de pesquisa e de assistência na promoção para uma vida saudável de pessoas com Doença Arterial Coronariana (DAC) e teve como objetivo destacar a relevância do método da Pesquisa Convergente Assistencial (PCA) utilizada como referencial metodológico na condução do referido estudo. A intenção é contribuir para o avanço no desenvolvimento deste método de pesquisa que vem sendo cada vez mais utilizado em pesquisas na área da saúde, especialmente na enfermagem, possibilitando a produção de novos conhecimentos e a reconstrução de práticas em saúde.

0 estudo desenvolvido com a utilização da PCA (neste artigo será denominado pesquisa de referência) teve 0 propósito de introduzir inovações no cuidado pela construção, implementação e análise de um Programa de Vivências Corporais Lúdicas (PVCL) com pessoas com DAC. ${ }^{1}$ A abordagem da PCA foi eleita como referencial metodológico na pesquisa de referência porque sua tipicidade exige ações conjuntas de pesquisa e de assistência de modo a direcionar o pesquisador para a resolução de problemas e ou introdução de inovações nas práticas de saúde, além de dar respaldo para construções teóricas expressivas ao cotidiano das situações pesquisadas. ${ }^{2}$

A abordagem da PCA, além de seu caráter de provocar mudanças, tem o potencial de responder a uma grande diversidade de questões de pesquisa, inclusive aquelas pertinentes às mudanças referentes às situaç̃oes crônicas de saúde, o que vem fortalecendo as travessias para o alcance de novos estilos de viver saudável. ${ }^{3}$

Na pesquisa de referência houve a intencionalidade de introduzir um inovador programa (PVCL) que consiste em práticas corporais lúdicas, que não somente incluem as atividades físicas, mas também consideram o sentido e as expressões dos movimentos baseados nas necessidades concretas de vida das pessoas. As vivências corporais lúdicas diferenciam-se dos programas tradicionais de prevenção e reabilitação de pessoas com DAC, uma vez que não seguem as mesmas características e os princípios do condicionamento físico quanto à intensidade e duração. Incluem atividades diversas tais como: expressão corporal, jogos, movimentos ginásticos, movimentos de alongamentos, exercícios de flexibilidade, ioga, massagem, danças, jogos, capoeira, tai-chichuan, hidroginástica e exercícios aeróbios. Para essas atividades, foram utilizadas diferentes técnicas e materiais, estilos e ritmos musicais. Deste modo, o PVCL caracteriza-se como um processo educativo em saúde que pode ser incorporado pelas pessoas como uma alternativa de promover saúde.

As condições crônicas têm tido impacto na qualidade de vida dos indivíduos e suas famílias. A literatura referente a esta problemática, de modo geral, mostra repetidas reflexões sobre a possibilidade de promoção de saúde e de prevenção das complicações crônicas. Estas são baseadas em duas posturas distintas: há uma vertente que defende a ideia de que a ocorrência das doenças crônicas está estreitamente associada ao estilo de vida que a pessoa leva e por isso ela é responsável pela sua condição crônica. Outra vertente não coloca a responsabilidade na pessoa, mas focaliza as condições individuais e coletivas para um viver saudável, que inclui condições dignas de habitação, educação, trabalho, enfim, possibilidade de tomar decisões saudáveis. Esta postura tem como sustentação a crença de que os governos têm o dever de apoiar as populações na melhoria de sua saúde e defende que a prevenção das doenças crônicas deverá iniciar com o apoio especial aos grupos vulneráveis como as crianças e a população de baixa renda. ${ }^{1}$ Até $80 \%$ das mortes por doenças crônicas acontecem em países pobres, pois as pessoas pobres têm escolhas limitadas sobre o que comem, suas condições de habitação, acesso à educação e cuidados de saúde. ${ }^{4}$ Dar apoio às escolhas saudáveis, especialmente para aqueles que de outro modo não poderiam fazê-las, reduz riscos e desigualdades. ${ }^{5} \mathrm{As}$ doenças cardiovasculares tornam-se comuns e são responsáveis pela maior causa de mortalidade no mundo. A DAC é a doença cardíaca de maior prevalência, que compromete a saúde do coração da grande maioria das pessoas na civilizações contemporâneas. ${ }^{6,7}$

Atualmente, os profissionais da saúde são solicitados a cuidar de pessoas em condições crônicas que, quando instaladas, já não podem ser prevenidas. 0 que fazer diante dessa situação? Pode-se, sim, prevenir a sua progressão por meio de uma educação ajustada às condições de vida de cada pessoa.

Nesse sentido, a assistência na pesquisa de referência teve como elemento o "lúdico", entendido como uma atividade livre, prazerosa, alegre, harmoniosa, acreditando que este pode levar as pessoas com DAC a se integrarem aos programas de prevenção e reabilitação cardiorrespiratória, objetivando desenvolver a criatividade, o prazer, a autonomia, a fantasia, entre outras manifestações; possibilitar melhor compreensão e percepção qualificada do movimento; e, por fim, oferecer oportunidades às pessoas para, ao (re)descobrirem o seu corpo, (re)descubram o significado e a importância de ser e estar no mundo. 0 lúdico se sente nas emoções, na excitação dos sentidos, no lado não racional, no lado não 'sério' do humano. ${ }^{8}$

A pesquisa de referência foi planejada com a convicção de que contribuiria para introduzir uma nova modalidade para a melhoria da saúde de um grupo de pessoas com DAC. Esta persuasão foi, em parte, ancorada nos pressupostos da Pesquisa Convergente Assistencial indicados pelas suas autoras:9:27-28

- 0 contexto da prática assistencial suscita inovação, alternativas de solução para minimizar ou solucionar problemas cotidianos em saúde e renovação de práticas em superação.

- 0 contexto da prática assistencial é potencialmente um campo fértil de questões abertas a estudos de pesquisa. 
- 0 espaço das relações entre a pesquisa e a assistência vitaliza simultaneamente o trabalho vivo no campo da prática assistencial e no da investigação científica.

- APCA implica no compromisso de beneficiar o contexto assistencial durante o processo investigativo, ao tempo em que se beneficia com 0 acesso franco às informações procedentes deste contexto.

- 0 profissional da saúde é potencialmente um pesquisador de questões com as quais lida cotidianamente, 0 que Ihe possibilita uma atitude crítica apropriada à crescente dimensão intelectual no trabalho que realiza.

\section{O CONTEXTO DA PESQUISA REFERÊNCIA}

As pessoas envolvidas na pesquisa de referência foram em número de 19, sendo 7 homens e 12 mulheres com doença arterial coronariana, com idades que variaram entre 51 e 82 anos. A Prática Assistencial consistiu na implementação do PVCL que ocorreu em uma sala de ginástica de um curso de graduação em educação física, onde foram realizados três encontros lúdicos semanais com duração de uma hora durante oito meses.

Na pesquisa de referência o pesquisador esteve com os participantes minutos antes do começo das vivências. Esses momentos que antecediam a atividade foram de grande importância na orientação e socialização das pessoas, estimulando-as a uma troca de relatos sobre suas vidas, famílias, saúde, doença, gostos, interesses e necessidades. A PCA permitiu a interação e a compreensão de particularidades da vida dos participantes.

A PCA adquire maior importância pelo seu caráter metodológico de proximidade e afastamento diante do saberfazer assistencial. Nesta proximidade e afastamento entre a PCA e a assistência, há permutas de recíprocas informações ao longo de ambos os processos: informações da pesquisa influenciando a prática assistencial em renovação e informações da prática alimentando as indagações processadas pela pesquisa. Esse movimento crítico constituiu uma ponte interativa e se mostra em claro delineamento, seja em momentos metodológicos, cuja dominância é a participação no cuidar em que o pesquisador envolvido está no cuidado, seja nos momentos em que o domínio maior é o da pesquisa, cuja dominância é metodológica. ${ }^{9}$

No início e no final de cada prática, o grupo ficava em pé ou sentado, formando um círculo, de mãos dadas, fazendo uma retrospectiva do encontro anterior, questionando como se sentiam naquele momento, estimulando a reflexão e socializando a proposta do novo encontro. Os princípios da PCA proporcionam aos participantes a reflexão sobre "o pensar e o fazer", possibilitando uma prática de assistência/cuidado em que o "fazer" inclui a objetividade e a subjetividade do participante em um processo reflexivo.
A PCA foi o tipo de pesquisa que permitiu utilizar diferentes técnicas de coleta de dados a partir da própria prática assistencial - vivências corporais lúdicas - juntamente com os depoimentos dos participantes, transformando-os em resultados da pesquisa. Considerando que a PCA permite a integração de vários métodos, estratégias e técnicas, o estudo foi realizado com a utilização de abordagem qualitativa, mas também utilizando abordagem quantitativa, especialmente na análise das mudanças anatomofisiológicas percebidas nos integrantes do programa. Essa possibilidade de integração de métodos e técnicas de coleta de dados foi uma das grandes vantagens no estudo efetuado.

Sendo assim, na pesquisa de referência foi realizada uma triangulação metodológica, que consistiu na combinação de diferentes métodos e procedimentos de coletas de dados de um mesmo estudo, com vistas a abranger, de forma mais acurada, as variadas perspectivas do objeto em estudo.

A observação foi o instrumento que incluiu as anotações no diário de campo, dos seguintes aspectos: descrição das pessoas, atitudes, olhares, comportamentos, palavras, atividades, medidas, enfim, tudo o que pudesse contribuir para desvelar o objetivo central da pesquisa. Esses registros constituíram os relatórios das Vivências Corporais Lúdicas que integraram o material de análise. Foram considerados 82 relatórios elaborados.

Outro procedimento de coleta de dados foi a entrevista semiestruturada, cujo objetivo foi aprofundar e validar alguns aspectos da observação envolvidos na temática do estudo. Este procedimento metodológico foi desenvolvido a partir da orientação de um roteiro com perguntas abertas buscando identificar e compreender as percepções que as pessoas com DAC experienciaram a respeito da saúde, doença, corpo, relacionamentos e vivências corporais. Como indica a PCA, os dados foram obtidos durante a prática assistencial. As pessoas aproveitaram esse momento para conversar, desabafar suas angústias, seus temores e incertezas diante das novas experiências vividas.

Foi realizada uma avaliação médica, incluindo anamnese, exame físico, exames laboratoriais, medidas de avaliação antropométrica, teste de esforço físico, medidas das dobras cutâneas e mensuração dos perímetros e da flexibilidade.

A principal característica da PCA consiste na sua articulação intencional com a prática assistencial. Dessa forma, as ações de assistência vão sendo incorporadas ao processo de pesquisa, o que não implica em atribuir idênticas características a estas duas atividades. Afinal, cada qual sustenta sua identidade própria, ou seja, tanto a pesquisa quanto a assistência têm as fronteiras delimitadas no que se refere à tipificação de conhecimento a que se vincula, aos aspectos éticos e ao rigor cientifico que lhe é pertinente. ${ }^{6}$ Durante o processo de estudo há momentos que as ações de pesquisa se sobrepõem às ações da prática assistencial e vice-versa, de modo a formar um movimento de aproximação entre as duas atividades, e há 
também momentos de afastamento formando um tipo de "dança". No ponto central nessa "dança" entre distanciamento e proximidade (pesquisa-assistência) é que fica respeitada a autonomia de cada um desses processos. ${ }^{4}$ Durante a realização da pesquisa, as semelhanças e diferenças entre tais processos tornam-se visíveis e a constituição da ponte entre eles é a base comum à construção do conhecimento novo dessa prática assistencial. $^{3}$

\section{A CONTRIBUIÇÃO DA PCA NO ESTUDO}

A abordagem da PCA mostrou-se relevante na condução da pesquisa de referência pelo montante de dados de pesquisa originados da convergência do processo de pesquisa e da implementação do PVCL, uma vez que a PCA permitiu ao pesquisador se envolver com atividades de pesquisa e assistência, possibilitando mudanças no modo de levar a vida de pessoas com DAC, a exemplo das mencionadas a seguir: ${ }^{1: 184}$ 185

- amenização dos momentos de solidão, propondo outros momentos de vida, de atividades ocupacionais, de lazer, sociais, artístico-culturais e físicas;

- aumento do bem-estar psicológico, reforçando a autoestima e autoconfiança e o conhecimento de si mesmas, com satisfação em relação ao corpo e aos sentimentos de realização e autoeficácia;

- diminuição dos níveis de tensão, ansiedade e depressão;

- contribuição como suporte de ajuda e de alternativas para que se adaptassem à nova realidade de conviver com uma doença crônica;

- rompimento com movimentos estereotipados e mecanizados para a prevenção e reabilitação, dando um novo sentido ao "se-movimentar";

- mudanças de comportamento na busca de conviver harmonicamente com a nova situação de ter uma doença crônica;

- troca de experiências, fazendo com que o grupo percebesse que existem outros problemas no mundo, e que eles não são os únicos que sofrem, levando-os ao crescimento pessoal.

Os achados de pesquisa incluíram informações sobre alguns aspectos da vida dos participantes tais quais: percepções em relação à doença e ao PVCL; desafios para enfrentar mudanças; dificuldades; receios; prazeres; motivações; amarras; conhecimento de si; relações e interações; sentimentos. Houve a constatação de mudanças anatomofisiológicas, incluindo: aumento da flexibilidade; variação no índice de massa corpórea e na circunferência abdominal; diminuição do colesterol total, dentre outras. Portanto, a abordagem da PCA deu abertura para criação do PVCL, para sua implementação e, de certa forma, para sua avaliação.
A PCA permitiu a interação entre pesquisadora e pesquisados no desenvolvimento de uma nova perspectiva de trabalhar com o movimento corporal, objetivando a prevenção e a reabilitação de pessoas com DAC, possibilitando construções de referenciais para a orientação dos profissionais da área da Saúde e da Educação Física, influenciando, assim, no processo de viver mais saudável dessas pessoas.

A proposta da prática desenvolvida proporcionou às pessoas com DAC um cuidar diferenciado dos programas tradicionais de prevenção e reabilitação cardíacas ao conceber: 10:20

as vivências corporais como um interrelacionamento entre vida, vivências e experiências, enfatizando que a sensibilidade, as percepções e as intuições do ser humano desenvolvem-se de forma mais aberta e intensa quanto maior for 0 grau e as oportunidades de vida, vivências e experiências com atividades constituídas por um se-movimentar espontâneo, autônomo e livre.

Assim, "o cuidar" não esteve compreendido no fazer por fazer, mas, sim, em articulação com as ações que envolvem pesquisadores e demais pessoas representativas da situação a ser pesquisada, em uma relação de cooperação mútua. ${ }^{5}$

Esse processo foi acompanhado de uma reflexão autocrítica e da avaliação dos resultados, tendo como finalidade contribuir simultaneamente com o alívio das preocupações de ordem prática das pessoas que estão em situação problemática. Visou-se a mudança na atitude dos participantes na relação com sua saúde, com sua doença, com seu corpo e com os outros.

Buscou-se, outrossim, contribuir para o desenvolvimento da ciência, produzindo um conhecimento que servisse de base para futuros trabalhos de profissionais que atuam com pessoas que apresentam doenças cardiovasculares, cujo propósito foi desenvolver experiências corporais alternativas às formas tradicionais que centram sua atenção no tratamento da doença, ignorando, muitas vezes, a pessoa portadora.

As vivências permitiram às pessoas com DAC experienciar o lúdico e a ludicidade, não só pela presença de brincadeiras ou jogos, mas também pelo envolvimento genuíno com as atividades, pela sensação de plenitude que transforma a prática do movimento em ação significativa. Também proporcionaram às pessoas um contato maior consigo mesmas e com os seus colegas, sentir o próprio corpo e o do seu companheiro, sentir os ritmos diferentes, perceber a sua expressão corporal e a de seu colega, enfim, permitiram expressar sentimentos de felicidade, satisfação, liberdade, sensibilidade, levando-os a sentir o prazer de estar "brincandocorporalmente juntos".

A elaboração desse estudo possibilitou novas metodologias na maneira de cuidar, de apreender e de ampliar conhecimentos por intermédio do PVCL para um viver mais saudável de pessoas com DAC. Os caminhos apresentados pela 
temática foram desafiadores. Propor alternativas de metodologias de trabalho enfatizando os movimentos corporais para a prevenção e reabilitação de pessoas com doenças cardiovasculares já expressa a dimensão de tais desafios.

A pesquisa de referência contribuiu também na busca de alternativas metodológicas a serem debatidas/desenvolvidas no processo de viver das pessoas com DAC, naformação dos profissionais da Saúde e da Educação Física, como, também, de outras áreas de conhecimento. Entendemos que os resultados obtidos podem estimular maiores reflexões sobre propostas teórico-metodológicas, que conduzam a um trabalho mais criativo, expressivo e comunicativo, e sustentar a tese proposta de que o PVCL oferece melhores condições para a pessoa com DAC se expressar, se manifestar por meio do movimento corporal, oportunizando construir possibilidades de se situar mais saudável no mundo. A busca de produção de mudanças como uma intencionalidade que agrega valor às novas práticas está presente na PCA, pelo andamento de suas estratégias. Destaca-se, na PCA como estratégia básica, a participação do pesquisador, tanto na investigação em si como na prática assistencial, de modo simultâneo, durante todo o processo de desenvolvimento da pesquisa. Este fortalecimento recíproco da PCA com a prática assistencial, a fim de gerar transformações e novos conhecimentos, consubstancia afinidades, quanto à intenção comum de gerar mudanças e compor novos e concretos modos de saber-fazer as ações de saúde.

\section{CONCLUSÕES}

A PCA configurou-se como um instrumento útil no desenvolvimento da pesquisa de referência por se revelar uma abordagem que articula em convergência teoria, pesquisa e prática assistencial e por possibilitar intervenções sintetizadas em mudanças.

Deste modo, os sujeitos da pesquisa foram beneficiados com uma melhor compreensão de suas problemáticas e com a descoberta de alternativas para lidar com elas. Os resultados mostraram que uma das maneiras eficientes de educar em saúde é incentivar a participação dos sujeitos no processo de pesquisa e que a interação deles e dos pesquisadores facilita uma maior perscrutação das suas condições de vida e de saúde.

0 estudo contribuiu na produção de conhecimento referente aos modos de cuidar de si e conviver bem com a doença crônica. Assim, a PCA mostrou ser um método apropriado para introduzir inovações nos modos de educação para o cuidado de si e na prevenção de possíveis complicações relacionadas a DAC.

Destacamos a importante contribuição da PCA na aproximação entre teoria e prática, o que tem sido considerado um desafio por vários profissionais da saúde. Esta articulação entre teoria e prática mantém-se pela exigência do pesquisador no campo, envolvendo-se diretamente com o objeto de investigação, porém, preservando os princípios e o rigor do método científico.

\section{REFERÊNCIAS}

1. Bonetti A. 0 coração e o lúdico: vivências corporais lúdicas para um viver mais saudável de pessoas com doenças aterosclerótica coronariana [tese de doutorado]. Florianópolis(SC): Programa de Pós-Graduação em Enfermagem/UFSC; 2006.

2. Trentini M, Paim L. Pesquisa em enfermagem: uma modalidade convergente-assistencial. Florianópolis(SC): UFSC; 1999.

3. Trentini M, Paim L. An innovative approach to promote a healthy lifestyle for persons in chronic conditions in Brazil. In: Turley AB, Hofmann GC. Life Style and health research progress. New York (EUA): Nova Publischer; 2008.

4. Organização Mundial da Saúde. Prevenção de doenças crônicas: um investimento vital. 2005 [citado em 20/4/2007]. Disponível em:<http:www.who.int/chip/chronic-disease-report>.

5. Organização Mundial da Saúde. Cuidados Inovadores para condições crônicas: componentes estruturais de ação: Relatório Mundial. Brasília(DF): OMS; 2003.

6. Smith LK, Brener S, Pashkow F. Intervervenções médicas e invasivas no tratamento da doença arterial coronariana - Manual de pesquisa das diretrizes do ACSM para os testes de esforço e sua prescrição. Rio de Janeiro(RJ): Guanabara Koogan; 2003.

7. SilvaSS, Caritá EC, Morais ERED. Fatores de risco para doença arterial coronariana em idosos: análise por enfermeiros utilizando ferramenta computacional. Esc. Anna Nery (online). 2010 Dez [citado 2011 Maio 02]; 14(4): 797-802. Disponível em: <http://www.scielo.br/ scielo.php?script =sci_arttext $\&$ pid $=$ S 1414 $81452010000400020 \&$ lng $=$ pt. doi: 10.1590/S1414$81452010000400020>$.

8. Santin, S. Educação Física: uma abordagem filosófica da corporeidade. ljuí (RS): Unijuí; 1987. (Coleção ensaios: política e filosofia)

9. Trentini M, Paim L. Pesquisa Convergente-assistencial: um desenho que une o fazer e o pensar na prática assistencial de saúde-enfermagem. $2^{\circ}$ ed. Florianópolis(SC): Insular; 2004.

10. Kunz E. Práticas didáticas para um "conhecimento de si" de crianças e jovens na Educação Física. In: Física 2. ljuí (RS): Unijuí; 2002. , organizador. Didática da Educação 